\title{
Interactions between CKD and MetS and the Development of CVD
}

\author{
Ken-ichi Aihara, ${ }^{1}$ Masaki Mogi, ${ }^{2}$ Rei Shibata, ${ }^{3}$ David Bishop-Bailey, ${ }^{4}$ and Xin L. Ma \\ ${ }^{1}$ Department of Medicine and Bioregulatory Sciences, The University of Tokushima, Graduate School of Health Biosciences, \\ 3-18-15 Kuramoto-cho, Tokushima 770-8503, Japan \\ ${ }^{2}$ Department of Molecular Cardiovascular Biology and Pharmacology, Ehime University Graduate School of Medicine, Shitsukawa, \\ Tohon, Ehime 791-0295, Japan \\ ${ }^{3}$ Department of Cardiology, Nagoya University Graduate School of Medicine, 65 Tsurumai, Showa-Ku, Nagoya 466-8550, Japan \\ ${ }^{4}$ Department of Translational Medicine and Therapeutics, William Harvey Research Institute, Barts and The London School of \\ Medicine and Dentistry, Queen Mary University of London, London EC1M6BQ, UK \\ ${ }^{5}$ Department of Emergency Medicine, Thomas Jefferson University, Philadelphia, PA 19107, USA
}

Correspondence should be addressed to Ken-ichi Aihara, aihara@clin.med.tokushima-u.ac.jp

Received 17 March 2011; Accepted 17 March 2011

Copyright ( 92011 Ken-ichi Aihara et al. This is an open access article distributed under the Creative Commons Attribution License, which permits unrestricted use, distribution, and reproduction in any medium, provided the original work is properly cited.

\section{Introduction}

Metabolic syndrome (MetS) consists of a combination of metabolic disorders, including increased abdominal circumference, hyperglycemia, elevated blood pressure, and lipid disorders. MetS is now widely accepted as a crucial risk factor for the development of cardiovascular disease (CVD) and mortality. In addition, persistent proteinuria indicating chronic kidney disease (CKD) is well known as a powerful risk factor for the progression of end-stage renal disease and CVD. In recent years, patients with CKD and MetS appear to be increasing along with increasing incidence of CVD in industrial countries.

In order to ameliorate cardiovascular mortality, it is essential to extend comprehensive treatments for patients with CKD and MetS. Although those disorders may be partly formed by a certain common pathological basis, there is insufficient knowledge of the underlying interplay between the pathological conditions and CVD. For this special issue, we asked front-line researchers and authors to submit original research and review articles on the interactions between CKD and MetS and the development of CVD. Finally, we were able to publish 10 review articles and 4 original research articles that provide pivotal evidence for understanding the pathophysiological relationships among the disorders on a clinical basis and a molecular basis. The following articles will serve very useful treatment strategies for promoting worldwide public health.

\section{Review Articles}

K. Nitta reviewed the epidemiology of MetS and CKD and focused on the possible linkage between CKD and MetS leading to an increase in cardiovascular events. The author described a fundamental treatment strategy including lifestyle modification and renal protection by renin-angiotensin blockade and/or statins.

D. Vykoukal and M. G. Davies showed influences of MetS on the hemostatic disorders and development of CKD and the outcomes of percutaneous renal intervention in patients with symptomatic atherosclerotic renal and fibromuscular dysplasia renal artery disease.

Y.-J. Sheen and W. H.-H. Sheu summarized the relation between MetS and CKD from epidemiological, biological, and clinical aspects. They clearly revealed the significance of several biomarkers and the importance of MetS as a therapeutic target for preventing CKD.

Since much attention has been paid to the role of oxidative stress in promoting not only the severity of CVD but 
also the progression of CKD, E. O. Gosmanova and N.-A. Le focused on the central roles of oxidative stress in the development of CVD and its complications in patients with CKD.

M. Raimundo and J. A. Lopes reviewed in detail the associations between and treatment strategies for CKD, MetS, and CVD. They clearly described clinical evidence and pathophysiology, including insulin resistance, inflammation, endothelial dysfunction, oxidative stress, renal circulation, renin-angiotensin-aldosterone system and sympathetic nervous system, and dietary factors.

Y. Beyazit et al. demonstrated the effect of bone marrow angiotensin II signaling on the initiation and progression of atherosclerosis with inflammation and oxidative stress involving endothelial cells and hematopoietic cells.

M. Mogi and M. Horiuchi showed the cerebrorenal connection mainly from a clinical point of view. They demonstrated that cerebral and glomerular small vessel diseases are based on a common pathogenesis through anatomic and vasoregulatory similarities. These issues indicate that CKD markers may be helpful for predicting the future risk of neuronal diseases such as stroke, dementia, and cognitive impairment.

K. Aihara et al. focused on the interplay between the TGF- $\beta 1$ signaling pathway and the development of CVD, $\mathrm{CKD}$, and MetS. Understanding the TGF- $\beta 1$ signaling pathway and appropriate modulation of the biological actions of TGF- $\beta 1$ is a valuable therapeutic approach to reduce CVD.

Y. Okamoto reviewed the clinical significance of hypoadiponectinemia in cardiovascular diseases and the protective features of adiponectin against cardiovascular remodeling, including cardiac hypertrophy, hypertension, atherosclerosis, and pulmonary artery hypertension.

M. Tesauro et al. showed that adipose tissue secretes bioactive adipokines, including adiponectin, leptin, adipsin, and visfatin, into the whole body circulation. Accumulating evidence about these factors would serve a strategy for protection of both renal and cardiovascular systems.

\section{Original Research Articles}

Since height is inversely associated with augmentation index indicating arterial stiffness, T. Takenaka et al. showed that not only cigarette smoking but also short height independently contributes to total mortality in hemodialysis patients.

V. B. Djordjević et al. showed that available biomarkers in patients with coronary artery disease with and without statin treatment are $\mathrm{C}$-reactive protein in acute myocardial infarction, triglyceride/apoB in stable angina pectoris, and lipoprotein (a) in unstable angina pectoris.

V. S. Rao et al. showed that among the biological cardiovascular risk markers, oxidized LDL cholesterol is the most powerful predictor of the incidence of MetS in 2316 individuals who were recruited in Phase I of the Indian Atherosclerosis Research Study (IARS).

C. S. Coffey et al. showed that subjects with MetS have higher levels of PAI-1 and t-PA antigen than do subjects without MetS in a population study. Since these factors have been known to increase the risk of cardiovascular disease, they raised the possibility that an accelerated coagulation state is linked with the pathological basis of MetS.

\author{
Ken-ichi Aihara \\ Masaki Mogi \\ Rei Shibata \\ David Bishop-Bailey \\ Xin L. Ma
}




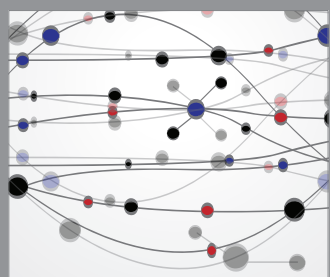

The Scientific World Journal
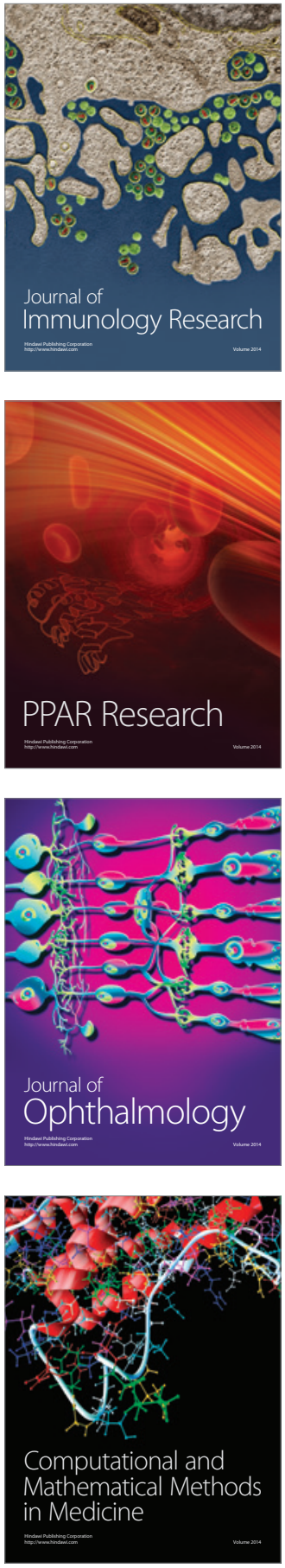

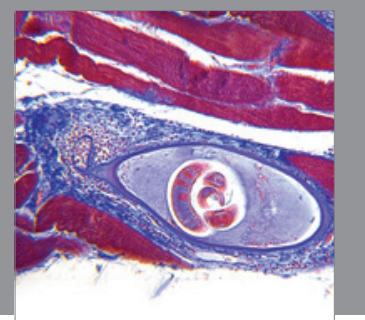

Gastroenterology

Research and Practice
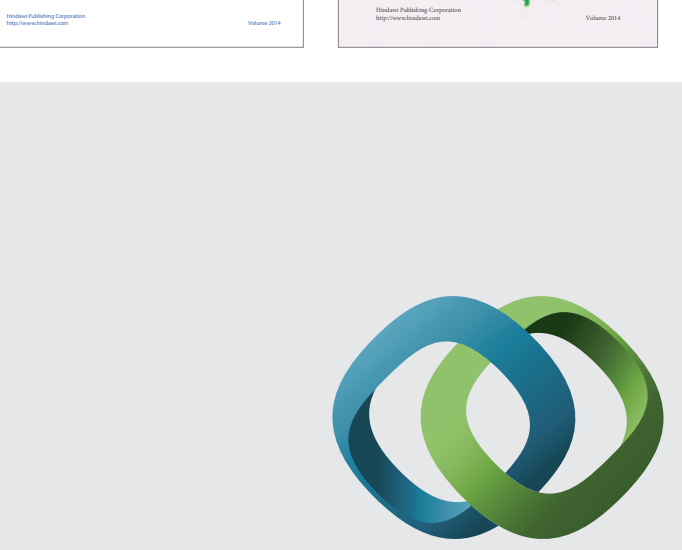

\section{Hindawi}

Submit your manuscripts at

http://www.hindawi.com
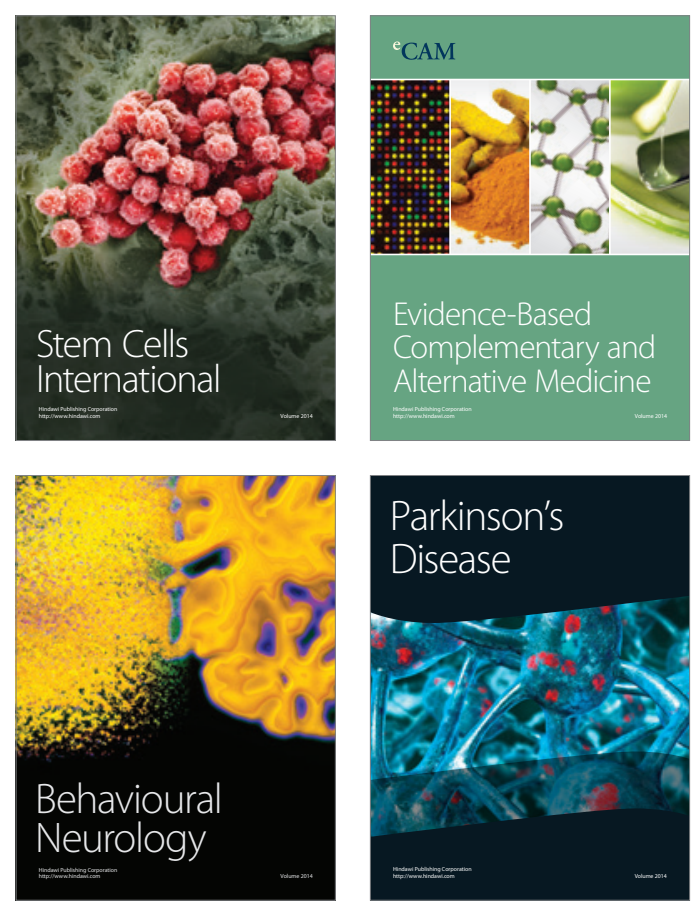

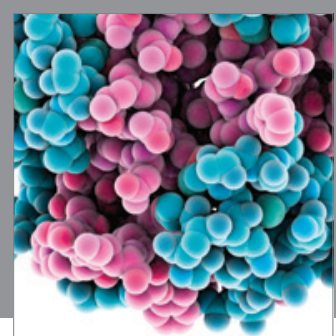

Journal of
Diabetes Research

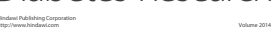

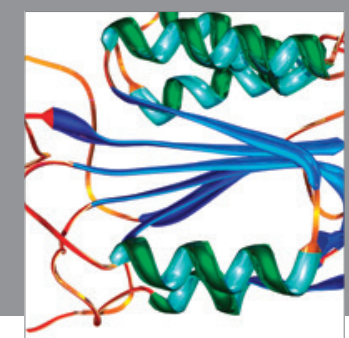

Disease Markers
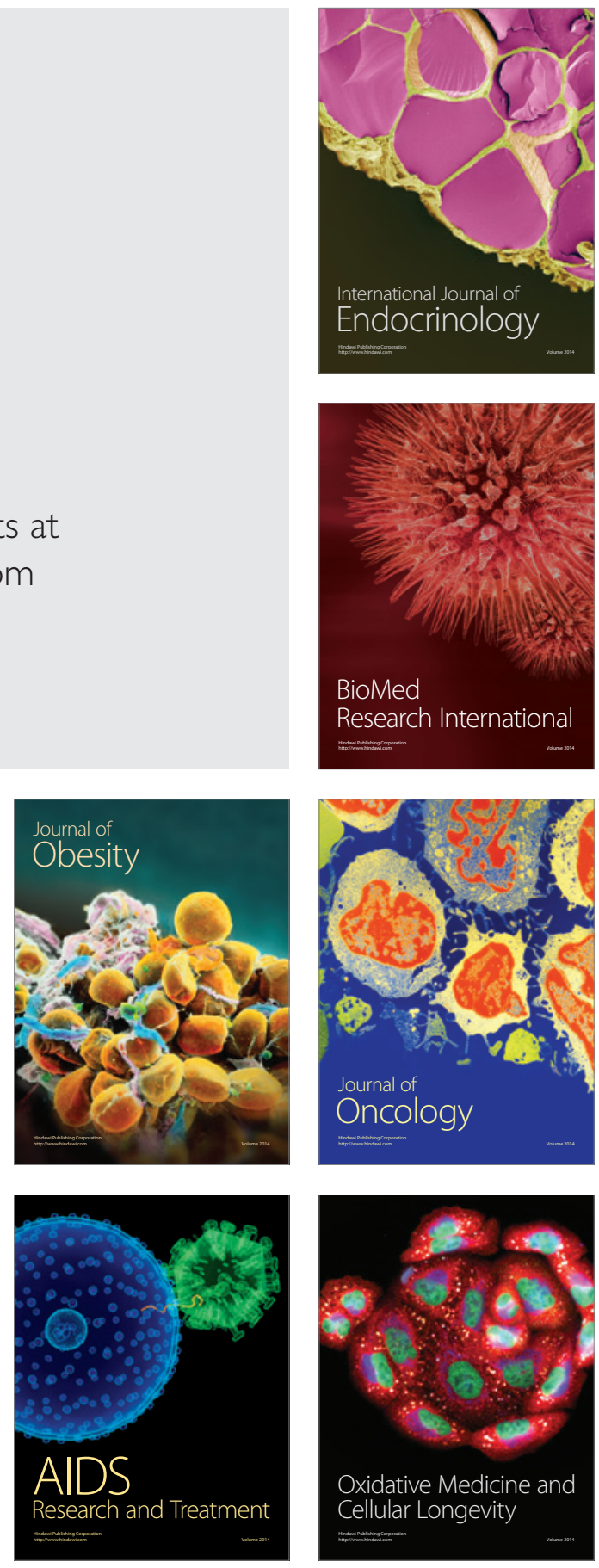